\title{
Dynamic Response Analysis of Spur Gear System with Backlash
}

\author{
Chao Li ${ }^{1, *}$, Jigang Wang ${ }^{1}$ \\ ${ }^{1}$ Department of Computer, Jiangsu Automation Research Institute, Lianyungang, China
}

\begin{abstract}
There are few studies on space-driven gear systems in the existing literature. In this paper, a spacedriven two-stage spur gear system is taken as the research object, and a 10 DOF dynamic model is established. A nonlinear dynamic response analysis was performed. The backlash was introduced into the dynamic model, and the time-varying stiffness was corrected to make the theoretical model closer to reality. By comparing two kinds of dynamic response curves with and without return difference, it was illustrated that the influence of return difference on dynamic transmission error in a gear system. The results obtained in this paper provide a reference and basis for subsequent research.
\end{abstract}

\section{Introduction}

For decades, the dynamics of gear systems has been receiving widespread attention. A lot of research has been done on the nonlinear vibration of the gear system based on the system's nonlinear vibration theory and nonlinear factors such as time-varying meshing stiffness, error excitation, damping, and backlash of the gear meshing process. [1,2] According to the number of nonlinear factors, the specific conditions of the gear system, and the purpose of analysis, various forms of dynamic models [35] have been proposed. Among them, the nonlinear dynamic model is the simplest analytical model, which mainly used to study the vibration characteristics generated by the gear meshing itself, which reflects that the gear system is a nonlinear parametric vibration system. In a certain sense, it is the nonlinear dynamic model that reflects the basic properties and characteristics of the dynamics of the gear system, and determines the theory and method that must be adopted for the analysis and research. Therefore, the nonlinear dynamic model has always been the core problem of gear dynamic system research. A large number of theories and experiments at home and abroad show that this model is effective in studying the dynamic characteristics of gears.

$$
\begin{aligned}
& m_{1} \ddot{y}_{1}=-k_{y 1} y_{1}-c_{y 1} \dot{y}_{1}+k_{t 1}\left(r_{b 1} \theta_{1}-r_{b 2} \theta_{2}-y_{1}+y_{2}\right) \\
&+c_{t 1}\left(r_{b 1} \dot{\theta}_{1}-r_{b 2} \dot{\theta}_{2}-\dot{y}_{1}+\dot{y}_{2}\right) \\
& m_{2} \ddot{y}_{2}=-k_{y 2} y_{2}-c_{y 2} \dot{y}_{2}-k_{t 1}\left(r_{b 1} \theta_{1}-r_{b 2} \theta_{2}-y_{1}+y_{2}\right) \\
&-c_{t 1}\left(r_{b 1} \dot{\theta}_{1}-r_{b 2} \dot{\theta}_{2}-\dot{y}_{1}+\dot{y}_{2}\right) \\
& m_{3} \ddot{y}_{3}=-k_{y 3} y_{3}-c_{y 3} \dot{y}_{3}+k_{t 2}\left(r_{b 3} \theta_{3}-r_{b 4} \theta_{4}-y_{3}+y_{4}\right) \\
& \quad+c_{t 2}\left(r_{b 3} \dot{\theta}_{3}-r_{b 4} \dot{\theta}_{4}-\dot{y}_{3}+\dot{y}_{4}\right)
\end{aligned}
$$

\footnotetext{
${ }^{*}$ Corresponding author: sansking @163.com
} 


$$
\begin{gathered}
m_{4} \ddot{y}_{4}=-k_{y 4} y_{4}-c_{y 4} \dot{y}_{4}-k_{t 2}\left(r_{b 3} \theta_{3}-r_{b 4} \theta_{4}-y_{3}+y_{4}\right) \\
\quad-c_{t 2}\left(r_{b 3} \dot{\theta}_{3}-r_{b 4} \dot{\theta}_{4}-\dot{y}_{3}+\dot{y}_{4}\right) \\
J_{1} \ddot{\theta}_{1}=k_{1}\left(\theta_{0}-\theta_{1}\right)+c_{1}\left(\dot{\theta}_{0}-\dot{\theta}_{1}\right)-r_{b 1}\left[k_{t 1}\left(r_{b 1} \theta_{1}-r_{b 2} \theta_{2}-y_{1}+y_{2}\right)\right. \\
\left.+c_{t 1}\left(r_{b 1} \dot{\theta}_{1}-r_{b 2} \dot{\theta}_{2}-\dot{y}_{1}+\dot{y}_{2}\right)\right] \\
J_{2} \ddot{\theta}_{2}=-k_{2}\left(\theta_{2}-\theta_{3}\right)-c_{2}\left(\dot{\theta}_{2}-\dot{\theta}_{3}\right)+r_{b 2}\left[k_{t 1}\left(r_{b 1} \theta_{1}-r_{b 2} \theta_{2}-y_{1}+y_{2}\right)\right. \\
\left.+c_{t 1}\left(r_{b 1} \dot{\theta}_{1}-r_{b 2} \dot{\theta}_{2}-\dot{y}_{1}+\dot{y}_{2}\right)\right] \\
J_{3} \ddot{\theta}_{3}=k_{2}\left(\theta_{2}-\theta_{3}\right)+c_{2}\left(\dot{\theta}_{2}-\dot{\theta}_{3}\right)-r_{b 3}\left[k_{t 2}\left(r_{b 3} \theta_{3}-r_{b 4} \theta_{4}-y_{3}+y_{4}\right)\right. \\
\left.+c_{t 2}\left(r_{b 3} \dot{\theta}_{3}-r_{b 4} \dot{\theta}_{4}-\dot{y}_{3}+\dot{y}_{4}\right)\right] \\
J_{4} \ddot{\theta}_{4}=-k_{3}\left(\theta_{4}-\theta_{5}\right)-c_{3}\left(\dot{\theta}_{4}-\dot{\theta}_{5}\right)+r_{b 4}\left[k_{t 2}\left(r_{b 3} \theta_{3}-r_{b 4} \theta_{4}-y_{3}+y_{4}\right)\right. \\
\left.+c_{t 2}\left(r_{b 3} \dot{\theta}_{3}-r_{b 4} \dot{\theta}_{4}-\dot{y}_{3}+\dot{y}_{4}\right)\right]
\end{gathered}
$$

\section{Effect of return difference on gear system}

\subsection{Calculation of return difference of transmission chain}

When the backlash is numerically synthesized, it can be known from the central limit theorem that the backlash after the factors affecting the backlash is necessarily a normal distribution. Obviously, the comprehensive return difference also conforms to the normal distribution.

When designing the gear transmission, consider the selection conditions of the center distance deviation value $f_{a}$, so that the accuracy of the hole distance on the box, the bearing clearance and the accuracy of other components that make up the gear pair are compatible with the accuracy grade of the gear.

At this time, the calculation formula of the gear pair hysteresis is:

$$
j_{\varphi}=\frac{6.878}{m_{n} z} \cdot 2\left[\left(E_{a m 1}^{\prime \prime}+E_{a m 2}^{\prime \prime}\right) \pm \sqrt{\left(\frac{E_{a 1}^{\prime \prime}}{2}\right)^{2}+\left(\frac{E_{a 2}^{\prime \prime}}{2}\right)^{2}+f_{a}^{2}}\right] \tan \alpha
$$

Assuming that the average backlash caused by the manufacturing factors of the gears in the above formula is, then

$$
\mu_{j_{E}^{\prime}}=E_{a m}^{\prime \prime} \cdot 2 \tan \alpha=\frac{E_{a s}^{\prime \prime}+E_{a i}^{\prime \prime}}{2} \cdot 2 \tan \alpha=\left(E_{a s}^{\prime \prime}+E_{a i}^{\prime \prime}\right) \tan \alpha
$$

The backlash limit deviation caused by gear manufacturing factors is

$$
L_{j_{E}^{\prime}}=\frac{E_{a}^{\prime \prime}}{2} \cdot 2 \tan \alpha=\frac{E_{a s}^{\prime \prime}-E_{a i}^{\prime \prime}}{2} \cdot 2 \tan \alpha=\left(E_{a s}^{\prime \prime}-E_{a i}^{\prime \prime}\right) \tan \alpha
$$

The combined formula of the backlash of the gear pair is

$$
j_{t}=\left(\mu_{j_{E_{1}}^{\prime}}+\mu_{j_{E_{2}}^{\prime}}\right) \pm \sqrt{L_{j_{E_{1}}^{\prime}}^{2}+L_{j_{E_{2}}^{\prime}}{ }^{2}+L_{f_{a}}{ }^{2}}
$$

The total return difference of the transmission chain is obtained by superimposing and combining the return differences of each pair of gears and transmission elements in the transmission chain. For gear reduction systems, the total return difference is generally calculated on the shaft of the driven wheel.

Figure 1 is a schematic diagram of the two-stage gear transmission system. The return difference of each pair of gears is marked on the driven shaft.

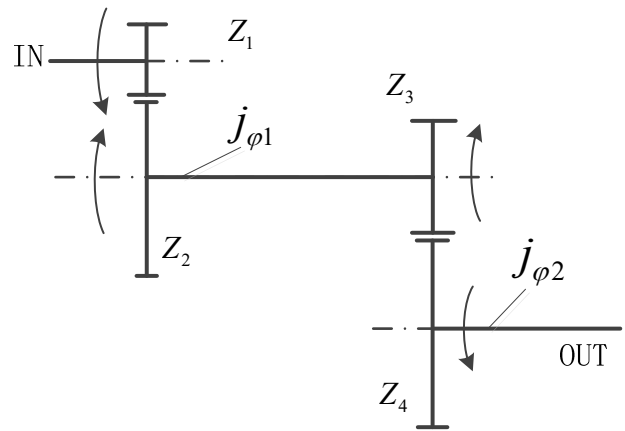

Figure 1 Schematic diagram for calculating the backlash of the transmission chain

The return difference of each pair of gears is converted to the output shaft, and then superimposed to obtain the return difference of the transmission chain of the twostage gear reduction system.

Maximum return difference

$$
j_{\varphi_{\max }}=5.842\left({ }^{\prime}\right)
$$

\subsection{Impact of Backlash on System Dynamic Response}

The dynamic model of the time-varying stiffness of the two-stage gear transmission with tooth profile and pitch error has been derived, and MATLAB programs have been written and verified using Adams software. The gears that mesh with each other are in constant contact meshing state because there is no gap, so their time-varying stiffness also exist from time to time. However, when factors such as backlash caused by return difference exist, the two gears that originally meshed with each other may not always 
maintain the meshed state, especially when the system is just starting. A small gear must mesh with a large gear after turning through the angle corresponding to the idle motion. At this time, the meshing stiffness starts to exist, so the equation of the gear torsion in the above dynamic equation needs to be modified. The modified mesh line stiffness is

$$
\begin{aligned}
& k_{t 1}^{\prime}=\left\{\begin{array}{l}
0, \theta_{1} \leq j_{\varphi 1} \\
k_{t 1}, \theta_{1}>j_{\varphi 1}
\end{array}\right. \\
& k_{t 2}^{\prime}=\left\{\begin{array}{l}
0, \theta_{3} \leq j_{\varphi 2} \\
k_{t 2}, \theta_{3}>j_{\varphi 2}
\end{array}\right.
\end{aligned}
$$

Change the restraint method between the load and the ground to the rotating pair, and add friction to the position of each rotating pair. The simulation time is set to $50 \mathrm{~s}$ and the number of simulation steps is set to 10,000 steps. Set the input angular velocity of the motor to $\omega_{0}=0.10472 \mathrm{rad} / \mathrm{s}$

. The response curve of the angular velocity of the first-stage pinion after simulation is shown in figure 2 , and the fluctuation points are marked in the figure.

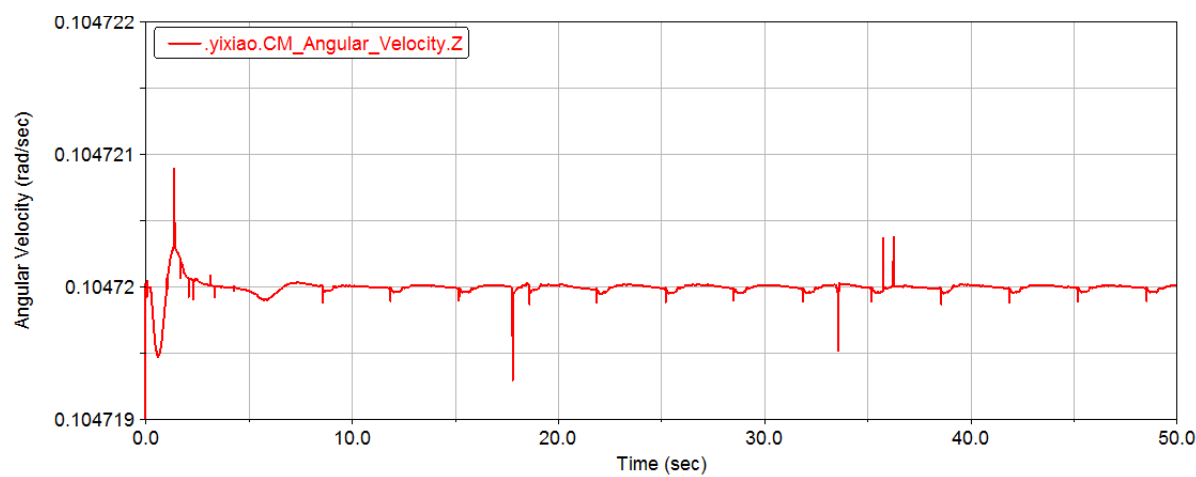

Figure 2 Curve of angular velocity of the first-stage pinion

The time points shown in the figure are as follows:

Table 1 Time table of points in the figure

\begin{tabular}{|l|l|l|l|l|l|l|l|l|l|}
\hline Point & 1 & 2 & 3 & 4 & 5 & 6 & 7 & A & B \\
\hline Time $(\mathrm{s})$ & 18.625 & 21.88 & 25.225 & 28.53 & 31.875 & 35.205 & 38.59 & 17.805 & 33.6 \\
\hline
\end{tabular}

From the table, it can be calculated that the time difference between any two adjacent fluctuation points of the speed fluctuation point is about $3.334 s$, and the time difference between the fluctuation points $A 、 B$ is about $15.795 s$. This time difference is basically consistent with the time of the speed impact point analysis.

The response curve at the load end is shown in figure
3. It can be seen that the load will still fluctuate around the average angular velocity $0.0010472 \mathrm{rad} / \mathrm{s}$ after a certain period of time. The fluctuation range is $0.001078 \mathrm{rad} / \mathrm{s}$ and exceeded average $2.94 \%$ as shown in the figure.

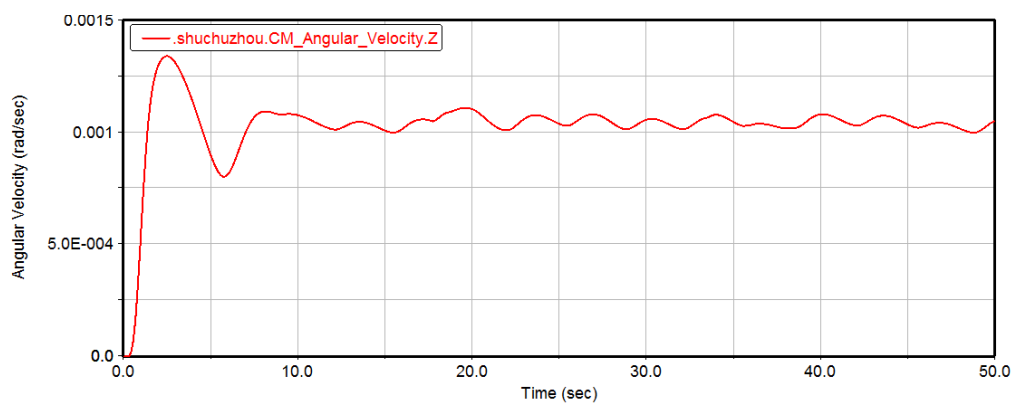

Figure 3 Angular velocity response curve of output load

Therefore, to facilitate the simulation, the constraint conditions in Adams are changed here. A fixed constraint is placed on the motor to fix the motor to the ground, and a torque is added to the load. The function expression of the torque $1 N \cdot \mathrm{m}$ is expressed as step (time, 0, 0,0.001,1) + step (time, 2, 0, 2.001,-1) . Within a period of time, a moment of magnitude is caused, each gear and load in the two-stage gear system generates a torsional displacement according to its own constraints, and then the torque is quickly reduced to 0 at time, in order to simulate passing way to see how the system responds under that condition. Other constraints remain the same. Set the simulation time to $15 \mathrm{~s}$ and the number of simulation steps to 30,000 . The following figure shows the load response curve with and without hysteresis. 


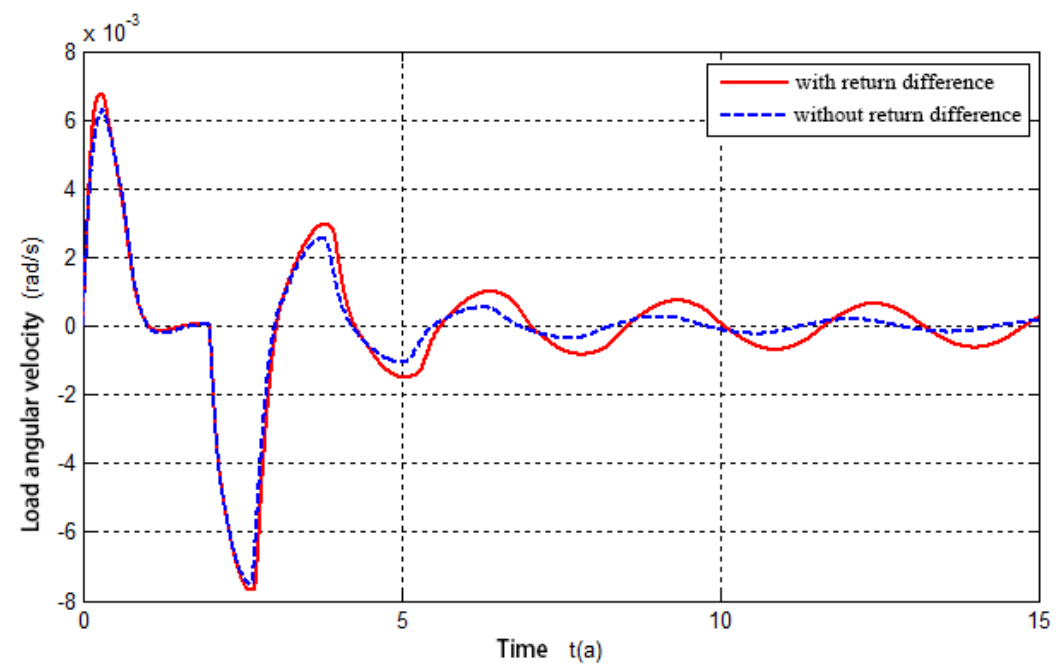

Figure 4 Load response curve of gear system with and without backlash under preload

It can be seen from figure 4 that the two sets of curves are obviously different. The response of the gear drive without hysteresis after the torque at the load end becomes 0 is faster than that of the gear system with hysteresis. The load of the gear system with hysteresis decays very slowly. After the torque becomes zero, there will be multiple oscillations, and the amplitude is larger than the amplitude of the shock of the gear system without hysteresis.

\section{Conclusion}

Taking the two-stage spur gear space-driven deceleration system as the research object, a 10-DOF nonlinear timevarying dynamic model was established. The ODE solver was used to solve the model based on the Runge-Kutta method using Matlab. The vibration acceleration curve of the periodic change of the gear system was obtained, and the correctness of the dynamic model was verified. The transmission error response curve exhibits time-varying characteristics, which is consistent with the change of time-varying stiffness.

This paper analyzes the relationship between return difference and backlash, and derives the theoretical formula between the two. The backlash was introduced into the dynamic model, and the time-varying stiffness was corrected to make the theoretical model more realistic. The research in this paper enriches the study on space drive systems and high load gear systems. The results obtained in this article provide a reference and basis for subsequent research.

\section{References}

1. Abboudi K, Walha L, Driss Y, et al. Dynamic behavior of a two-stage gear train used in a fixedspeed wind turbine $[\mathrm{J}]$. Mechanism and Machine Theory, 2011,46(12):1888-1900.

2. Hammami A, Fernandez Del Rincon A, Chaari F, et al. Effects of variable loading conditions on the dynamic behaviour of planetary gear with power recirculation[J]. Measurement, 2016,94:306-315.

3. Shen Y, Yang S, Liu X. Nonlinear dynamics of a spur gear pair with time-varying stiffness and backlash based on incremental harmonic balance method $[\mathrm{J}]$. International Journal of Mechanical Sciences, 2006,48(11):1256-1263.

4. Yang T, Yan S, Han Z. Nonlinear model of space manipulator joint considering time-variant stiffness and backlash[J]. Journal of Sound and Vibration, 2015,341:246-259.

5. Rao Z, Zhou C Y, Deng Z H, et al. Nonlinear torsional instabilities in two-stage gear systems with flexible shafts[J]. International Journal of Mechanical Sciences, 2014,82:60-66. 